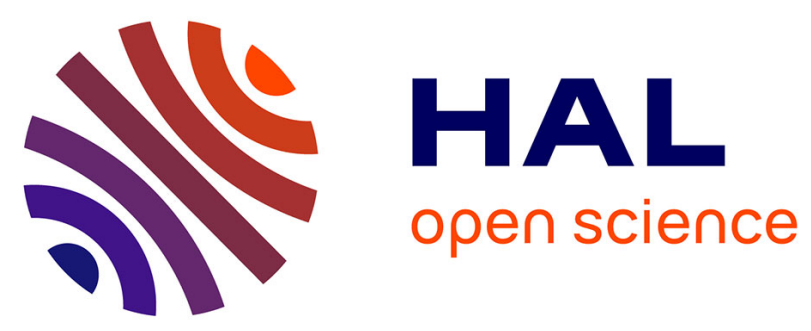

\title{
Opportunistic Alert Diffusion in Disaster Scenario -Stay Alive Longer !
}

Farouk Mezghani, Nathalie Mitton

\section{To cite this version:}

Farouk Mezghani, Nathalie Mitton. Opportunistic Alert Diffusion in Disaster Scenario -Stay Alive Longer!. IEEE PIMRC 2017 - The 28th Annual IEEE International Symposium on Personal, Indoor and Mobile Radio Communications, Oct 2017, Montreal, Canada. hal-01572088v2

\section{HAL Id: hal-01572088 \\ https://hal.inria.fr/hal-01572088v2}

Submitted on 19 Oct 2017

HAL is a multi-disciplinary open access archive for the deposit and dissemination of scientific research documents, whether they are published or not. The documents may come from teaching and research institutions in France or abroad, or from public or private research centers.
L'archive ouverte pluridisciplinaire HAL, est destinée au dépôt et à la diffusion de documents scientifiques de niveau recherche, publiés ou non, émanant des établissements d'enseignement et de recherche français ou étrangers, des laboratoires publics ou privés. 


\title{
Opportunistic Alert Diffusion in Disaster Scenario - Stay Alive Longer !
}

\author{
Farouk Mezghani, Nathalie Mitton \\ Inria Lille Nord - Europe, France - \{farouk.mezghani, nathalie.mitton\}@inria.fr
}

\begin{abstract}
Opportunistic communications present a promising solution as a disaster network recovery in emergency situations such as hurricanes, earthquakes and floods where infrastructure might be damaged. Recent works have proposed opportunistic-based disaster recovery solutions. However, two main features were left behind. On the one hand, these works do not consider the assortment of networks integrated in mobile devices (e.g. WiFi-Direct, WiFi ad-hoc, bluetooth). Moreover, they do not consider mobile devices that come with various energy levels. This paper proposes COPE, a cooperative opportunistic alert diffusion approach for disaster scenario useful for trapped survivors. COPE considers mobile devices equipped with multiple network interfaces and have various battery power levels. In order to maintain mobile devices alive longer, survivors form cliques and zones in which they diffuse alternately and periodically alert messages until reaching a potential rescuers. Simulation results show that COPE largely outperforms the selfish diffusion scheme in terms of energy consumption while guaranteeing an important alert delivery success.
\end{abstract}

\section{INTRODUCTION}

During disaster scenarios such as hurricanes, earthquakes and floods, communication is mostly needed for rescue operations of trapped survivors. However, network infrastructure might be damaged and thus no longer available making mobile communication devices such as smartphones, tablets and mobile phones affording practically no help.

Opportunistic communication has been investigated as a promising solution to partially overcome this problem [1], [2]. Survivors trapped inside a disaster area could use their mobile devices (e.g. smartphones) and exploit opportunistic communication to send emergency alert to proximate rescuers. However, alert diffusion in a disaster scenario presents different challenges. On the one hand, mobile devices come with multiple network interfaces (e.g. WiFi direct, WiFi adhoc, bluetooth) characteristics (e.g. coverage, throughput, energy consumption) and the choice is usually left to the user who has no idea what is best or might be in a physical or psychological distress preventing him/her from making this choice [3]. On the other hand, rescue operations may take long time, requiring mobile devices battery to be preserved as long as possible. Literature works have proposed opportunistic communication for alert

This work was partly supported by a grant from CPER DATA and PIPA project with SPOTROTTER partner.

978-1-5386-3531-5/17/\$31.00 (c) 2017 IEEE diffusion in disaster scenario [4-7] and all consider only one network interface. Most of these approaches rely on a selfish-based alert diffusion which might not be energy-efficient. Furthermore, they do not consider the various energy levels that will obviously impact the alert diffusion scheme since low energy level nodes cannot live as long as those with high energy level.

The aim of this paper is thus to design an opportunistic alert diffusion scheme for disaster scenario that exploits the multiple network technologies available in mobile devices and takes various battery levels into account. It presents COPE, an opportunistic cooperative alert diffusion scheme for emergency situations. COPE targets to rapidly reach proximity rescuers while maintaining devices alive for longer time. COPE performances are evaluated through extensive simulations over energy consumption and alert delivery success. Results show that COPE significantly outperforms the selfish-based diffusion in terms of energy consumption while guaranteeing an important alert delivery success.

This paper proceeds as follows. Section II presents the system model and the COPE scheme. In Section III, intensive simulation results demonstrate the effectiveness of COPE. Section IV reviews the related works. Finally, Section V presents the conclusion and future works.

\section{CoOperative Alert Diffusion}

System model: This work considers a set of nodes $\mathcal{U}=\left\{u_{i}\right\}$, each equipped with a mobile device characterized by an energy level and using multiple network interfaces $\mathcal{N}=\left\{n_{i} \mid i \in[1 . . N]\right\}$. These latter have different characteristics, mainly the energy consumption $(E C)$ and the transmission range $(T R)$ considered as the most important features in a disaster scenario. It is assumed that, for all $i \in[1 . . N-1]$ network interfaces, the $n_{i+1}^{t h}$ has a larger transmission range $(T R)$ and consumes more battery power $(E C)$ than network interface $n_{i}$ as follows: $T R_{n_{i+1}}>T R_{n_{i}} \& E C_{n_{i+1}}>E C_{n_{i}}$.

We emphasize that this model can also be suitable for a mobile network composed of nodes having each a single communication interface that can be managed by different transmission powers (i.e. low, medium, high) leading consequently to different transmission ranges/energy consumption. COPE dynamically copes with all kinds of devices and interfaces, making decision only on link characteristics. The transmission speed is not of great importance since alert diffusion messages are supposed of very short size requiring a normal 
transmission speed. The alert message represents a short message that comprises mainly the node identifier (ID) and location information to ease the rescue operation.

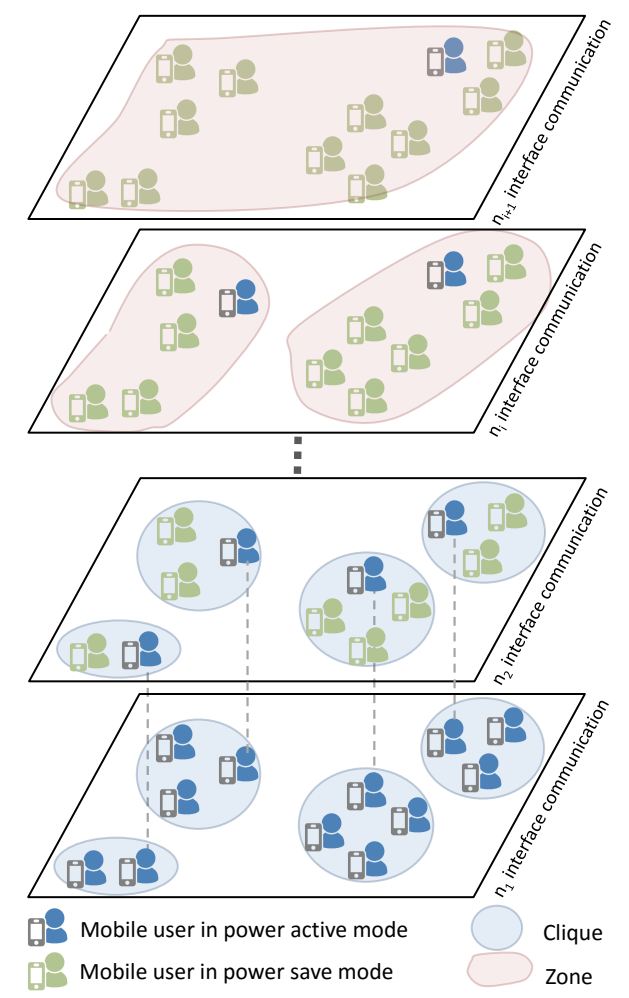

Fig. 1. Layer-based communication overview

COPE scheme: This work proposes COPE, an energy-aware Cooperative OPportunistic alErt diffusion scheme for disaster scenarios. COPE aims to help survivors coping with disaster scenarios by diffusing alert messages and preserving mobile devices battery as long as possible. COPE is mainly based on the following features. As survivors are usually trapped in groups during disaster scenarios, a cooperative-based diffusion to increase the battery power lifespan is considered. It performs an equity-based cooperation allowing nodes with low energy level to stay alive for longer time. Moreover, it deals with mobile devices that come with an assortment of networks and aims to perform a systematic network interface selection.

This work is based on a network layer-based communication scheme as shown in Fig. 1. Each layer presents the communication from the network interface $n_{i}$ perspective. The time horizon is divided into timeslots $\tau$. A time synchronization is required between nodes which is already done since mobile devices get the local time from the network providers with millisecond accuracy before disasters occur. As $\tau$ is at second level, no additional synchronization is required.

Nodes keep constantly the less-energy-consuming network interface $n_{1}$ active and use it to discover neighboring nodes and exchange their 1-hop neighbors. Hence, proximate nodes can form cliques. Exchanged messages contain information about the nodes ID and their energy level. From $n_{2}$ interface perspective, inside each clique, nodes cooperate, alternately, to discover neighboring nodes and diffuse the emergency alert. Hence, at an instant of time, only one node enter in active mode and diffuse the alert message using the interface $n_{2}$ while other nodes inside the same clique enter in sleep mode.

Nodes might initially have various energy-level. Therefore, a power threshold $p_{t h}$ is defined to determine the nodes that have a high energy level comparing to others. These latter will participate for a period of time two times higher than that of other nodes. Thus until making an energy balance between nodes. Based on the number of nodes inside a clique and their energy level, a time slot is divided in different periods of time during each only one node is in active mode (i.e. wake-up). The wake-up schedule of a node is determined in a distributed manner such as based on the node ID (e.g. node with the lowest ID starts diffusing first). If a node belongs to more than one clique, it computes its wakeup schedule considering the clique having the minimum number of nodes. Then, it informs nodes belonging to the same cliques about its wake-up schedule to take it into consideration.

If a node discovers other proximity nodes using the network interface $n_{2}$, these latter form a zone comprising the cliques that the nodes belong to. Afterwards, these nodes diffuse the zone information to the cliques they belong to, using interface $n_{1}$. Thereafter, nodes inside the same zone cooperate alternately for longer range diffusion using the network interface $n_{3}$. Following this methodology, from the $n_{i}^{t h}$ communication perspective, nodes inside the same zone cooperate alternately to discover other proximate zones and to alert potential proximity rescuers. If ever a node discovers other nodes from another zone, they form a superior zone and inform other nodes belonging to the same clique/zone using the active interfaces. Then, a cooperation inside the new zone is performed based on the network interface $n_{i+1}$.

When a survivor gets a response, s/he automatically inform the rescuer about other proximity cliques/zones to speed up rescue operations. It is assumed that the network topology is slowly time varying and update messages are exchanged when a node joins or leaves a clique/zone.

Motivating scenario and COPE overview: Fig. 2 illustrates a simple scenario featuring 7 nodes equipped each with a mobile device having 3 network interfaces $n_{1}, n_{2}$, and $n_{3}$ providing, respectively, low, medium and high transmission ranges and corresponds to low, medium and high battery power consumption. It considers survivors trapped in two proximate locations and can form two different cliques $C_{A}=\left\{s_{1}, s_{2}, s_{3}, s_{4}\right\}$ and $C_{B}=\left\{s_{5}, s_{6}, s_{7}\right\}$ using network interface $n_{1}$. 


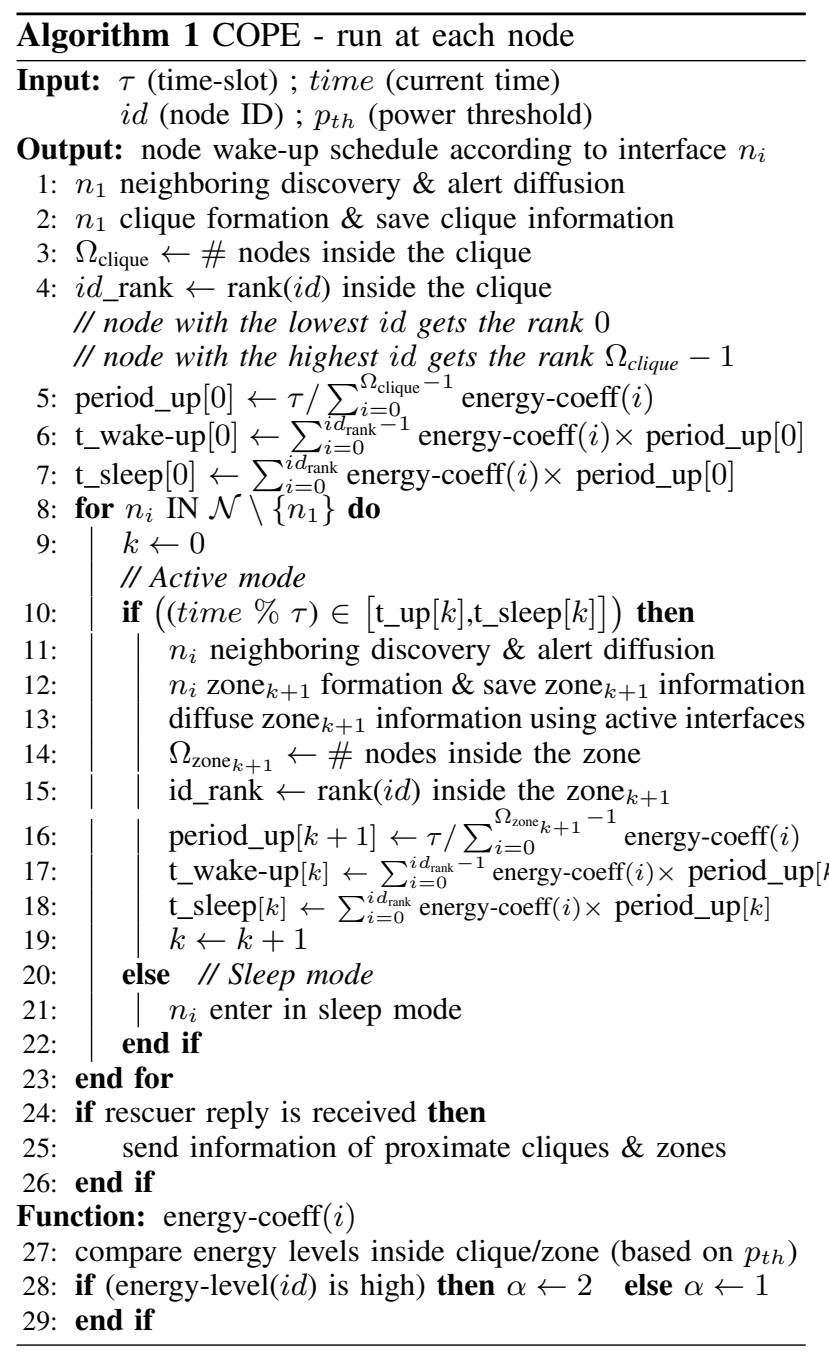

Let's assume that nodes have equal energy level except $s_{5}$ has a high battery power level. Inside clique $C_{A}$, using network interface $n_{2}$, nodes cooperate alternately to diffuse the alert message during a wake-up period of $\tau / 4$. On the contrary, nodes belonging to clique $C_{B}$ diffuse the alert message during a wake-up period of $\tau / 4$ except node $s_{5}$ for a wake-up period of $2 \tau / 4$ since it has a high energy level. Assuming that nodes $s_{1}$ and $s_{5}$ could discover each other using the interface $n_{2}$. Hence, they form a zone comprising cliques $C_{A}$ and $C_{B}$ and they diffuse the zone information to the nodes inside the same clique using interface $n_{1}$. Similarly, based on interface $n_{3}$, nodes inside the formed zone cooperate alternately for the alert diffusion during a wake-up period of $\tau / 8$ each except $s_{5}$ for $2 \tau / 8$.

This simple scenario shows the considerable energy saved compared to the selfish-based diffusion. Indeed, nodes belonging to cliques $C_{A}$ and $C_{B}$ can save, respectively, approximately $75 \%$ and $66 \%$ ( $85 \%$ respectively) of battery power, with respect to $n_{2}$ ( $n_{3}$ respectively) communication.
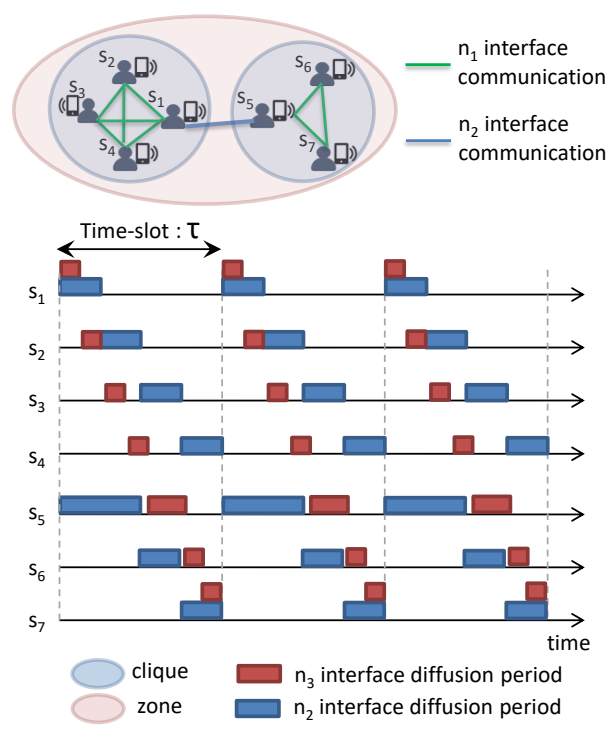

Fig. 2. Simple scenario

COPE Algorithm: Algorithm 1 gives the pseudocode for the COPE alert diffusion scheme run at each node. It describes how nodes cooperate and switch alternately between the active and sleep modes in a distributed way from the different network interfaces perspective.

First, nodes use the least powerful network interface for neighboring discovery and alert diffusion (L. 1). Neighbors form cliques and each node save information (i.e. neighbors ID and energy level) of its cliques allowing to determine the number of nodes inside a clique and to compute its rank among them (L. 2-4). According to the nodes energy-level and to their ID ranks inside the clique, each node computes its wakeup period duration and its schedule during the timeslot (i.e. diffusion start and end times) (L. 5-7). Nodes with high energy level are considered to participate twice more than other nodes with lower energy level (L. 27-30). During its active mode (L. 10 i.e. wake-up period), a node discovers neighboring nodes and diffuses the alert message based on the network interface $n_{i}$ (L. 11). Then, it forms a zone with neighbors from other cliques/zones and it sends the new zone information to other nodes belonging to the same cliques/zones using active interfaces (L. 12-13). Each node can thus determine its wake-up schedule inside the formed zone according to its ID and energy-level among those of other nodes inside the same zone (L. 14-18). During the sleep mode, a node simply deactivates the network interface $n_{i}$ and preserves its energy (L. 20-21). If a node gets an alert reply from rescuers, it sends the saved positions of proximate cliques and zones (L. 24-26) in order to speed up the rescue operations.

\section{PERFormance EVAluation}

COPE performances have been evaluated by simulations conducted through the Opportunistic Network Environment (ONE) [8]. Simulations involve a number 
of 35 mobile users considered as survivors. The mobility generator of BonnMotion has been used to generate mobility traces of users in disaster scenario [9]. The BonnMotion disaster mobility model generates movement driven by tactical reasons based on a method called separation of the rooms. Using this method, the disaster scenario is divided into different context-based areas which are : incident site, casualty treatment area, transport zone, and technical operational command zone. Conducted scenario considers a disaster area comprising 7 incident locations (e.g building, parking, restaurant) in which trapped survivors are randomly distributed waiting for help. We emphasize that survivors are considered with low mobility inside the incident locations. Thus, the network topology is considered slowly time varying.

Simulation scenarios consider users equipped with a mobile device having 3 network interfaces corresponding to low, medium and high transmission ranges and to low, medium and high battery power consumption. For the sake of simplicity, the energy level is expressed as a percentage of the battery capacity (i.e. 100\%: battery fully charged; $0 \%$ : battery empty). Mobile devices are considered with various initial energy level, each chosen randomly in the range of $[50 \%, 100 \%]$.

Cooperative vs Selfish diffusion - energy efficiency: COPE is compared with selfish and clique-based cooperative alert diffusion schemes. The former considers that each survivor only counts on himself for his survival. The survivor can either use many network technology of his mobile devices or s/he can use the most useful ones (e.g. interfaces with highest transmission range). The latter consists of cooperative diffusion limited to the nodes inside a clique formed by proximity nodes (without zone formation). Fig. 3 shows the average power consumption over time. Selfish based diffusion methods drain rapidly the batteries. Indeed, considering 2 and 3 network interfaces, the battery drains after approximately $3 \mathrm{~h} 30$ and 4 hours, respectively, which might not be efficient in practice since rescue operation can take a long time. Differently, cooperative-based alert diffusion schemes increase significantly the battery lifetime. Indeed, considering COPE and clique-based cooperative diffusion, the battery holds up to approximately 10 and 12 hours, respectively. This shows also that batteries can have longer lifetime when cooperating inside zones formed based on long-range communication.

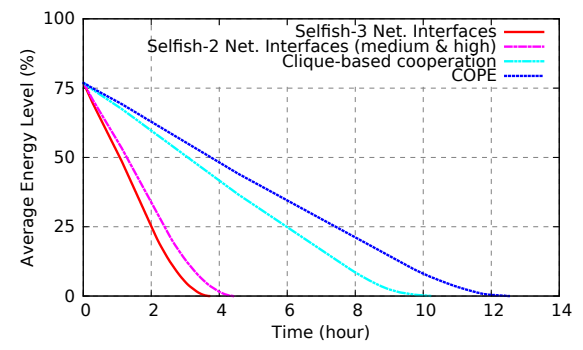

Fig. 3. Average energy level over time
Various initial energy levels: Considering different initial energy levels, we compare between COPE and equality-based cooperative alert diffusion. Unlike COPE, Equality is energy independent and considers an alert diffusion for equal period of time within the same clique/zone.

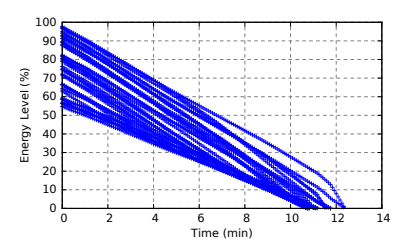

(a) COPE

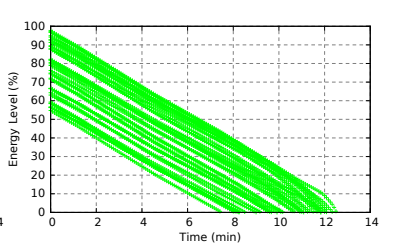

(b) Equality
Fig. 4. Energy consumption for each node over time

Fig. 4 presents the energy level of each node over time. It is clearly shown that COPE allows nodes with low energy to preserve their batteries for longer time comparing to equality-based diffusion. Fig. 5 presents the number of alive nodes (i.e. still have power in their batteries) over time. On the one hand, since nodes have initially various energy levels (difference up to $50 \%$ ), equality-based method allows the nodes with high energy to live for a long time (approximately 12.5 hours) while other nodes with low energy can live for much less time (approximately 8 hours). On the contrary, considering COPE, nodes with low energy can live longer (more than 10.5 hours). Obviously, nodes with high energy could live less time comparing to equalitybased method since they will spend more energy due to the longer diffusion period. In spite of that, these nodes wasted only few minutes ( $\sim 30 \mathrm{~min}$ ) which could maintain many other nodes in the network to stay alive longer (more hours) and consequently to maintain the whole network connected for longer time.

Alert message delivery efficiency: We now evaluate the alert delivery success. Simulations consider 100 different scenarios where a rescue-node moves with random path around and inside the disaster area. We count the successful emergency alert that could be delivered to the rescuer-node considering COPE, Equality-based and selfish (using 3 interfaces) alert diffusion methods. For a fair comparison, we consider a first scenario in which all nodes are alive ( $[0,1 h 30]$ on Fig. 3$)$.

Fig. 6 shows that the different diffusion methods succeed to deliver the alert message to the rescuer $(100 \%)$ for a walk and running speed $(1-1.5 \mathrm{~m} / \mathrm{s}$ and $2-4 \mathrm{~m} / \mathrm{s})$. When the rescuer speed increases $(6-8 \mathrm{~m} / \mathrm{s}$ and $12-14 \mathrm{~m} / \mathrm{s}$ ), Selfish diffusion can always deliver the emergency alert while COPE and Equality show a slow decrease since rescuer-node can enter and leave the coverage of a sleep-node before its wake-up. This case is not very realistic since in real environment rescuer-nodes will move slowly to carefully search for any survivor. Even though, COPE can be adapted by reducing the time-slot allowing nodes to switch quickly between the 


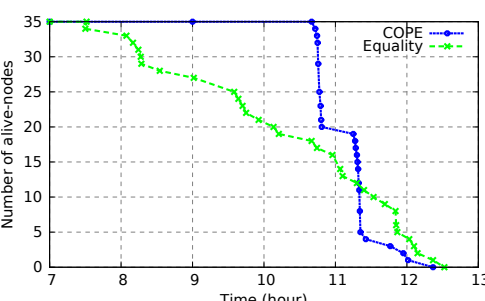

Fig. 5. Number of alive-nodes over time

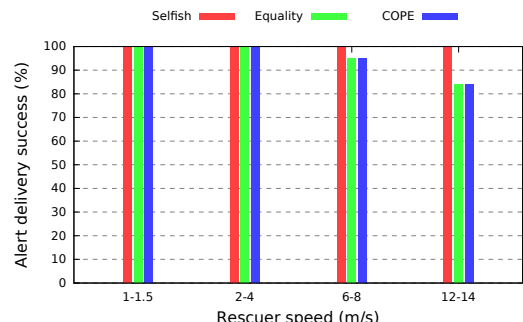

Fig. 6. Emergency alert delivery success (all nodes are alive)

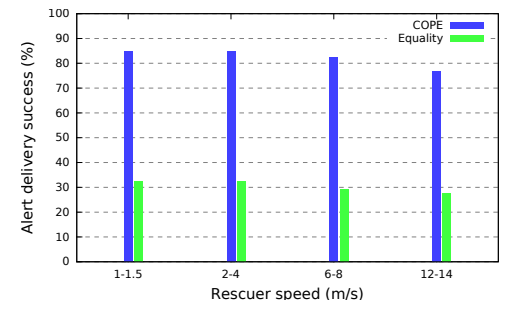

Fig. 7. Emergency alert delivery success (some nodes are alive) sleep and active modes.

The same scenario was performed for the period of time $[7 h, 12 h]$ (see Fig. 5) where only some nodes have energy left. Fig. 7 show the impact of the various initial energy levels on alert message delivery. COPE largely outperforms (more than $75 \%$ of delivery success) Equality (less than $35 \%$ of delivery success) since it takes account of the energy level difference between nodes and thus enables nodes with low energy to stay alive for longer time. Therefore, the network formed can perform a long time coverage of the disaster area and therefore increase the possibility to reach a rescuer moving in proximity. Considering Equality, the batteries of nodes with low energy will drain quickly and thus the network coverage is reduced and so the communication opportunity between the rescuer and alive-nodes.

\section{RELATED WORKS}

Several research works proposed solutions that seek to improve the disaster recovery, rescue operation, and emergency evacuation. Some literature works [4], [6] evaluate the performance of reference routing algorithms such as Epidemic and MaxProp and study their applicability to important tasks in disaster relief operations. Works in [1], [10] exploit opportunistic communication to collect message in disaster scenario and inform mobile users of emergency information such as impassable and congested roads to ease their evacuation. A multi-hop device-to-device communication scheme was proposed in [2] and uses smartphones to relay message in disaster area. [5] proposes a cooperative alert diffusion exploiting opportunistic communication allowing to minimize the energy consumption.

Even though many research works have contributed to improve disaster recovery and rescue operations, some of the picture is still missing. The aforementioned works have considered mobile devices equipped with only one network technology. However, mobile devices might have multiple network technologies. Furthermore, these works do not consider mobile devices that come initially with various energy levels. This works focus on opportunistic alert diffusion useful for trapped survivors to ease and speed up rescue operations. It differs from previous studies in that it considers the assortment of networks and is based on an automatic and systematic network interface selection. Additionally, this work is based on cooperative diffusion and takes into account various energy levels. Thus to preserve battery power as long as possible and maintain mobile devices with low battery level alive longer and so the network coverage.

\section{CONCLUSION}

This paper investigates the alert diffusion in disaster scenarios. COPE, a novel cooperative alert diffusion scheme, was proposed. Unlike existing works, COPE leverages on multiple network technologies integrated in mobile devices. COPE allows battery preservation for longer time and guarantees a high alert delivery success. The efficiency of COPE is emphasized through simulation studies and results show the significant performance of COPE comparing to individual alert diffusion. A proof-of-concept implementation to demonstrate the feasibility of our solution is a focus of our future work together with the investigation of the best rescuer path.

\section{REFERENCES}

[1] A. Fujihara and H. Miwa, "Disaster evacuation guidance using opportunistic communication: The potential for opportunitybased service," in Big Data and Internet of Things: A Roadmap for Smart Environments, 2014, pp. 425-446.

[2] H. Nishiyama, M. Ito, and N. Kato, "Relay-by-smartphone: realizing multihop device-to-device communications," IEEE Communications Magazine, vol. 52, no. 4, pp. 56-65, 2014.

[3] S. Deng, A. Sivaraman, and H. Balakrishnan, "All your network are belong to us: A transport framework for mobile network selection," in Workshop on Mobile Computing Systems and Applications (HotMobile). ACM, 2014, p. 19.

[4] D. Reina, M. Askalani, S. Toral, F. Barrero, E. Asimakopoulou, and N. Bessis, "A survey on multihop ad hoc networks for disaster response scenarios," Int. J. of Distributed Sensor Networks, vol. 2015, p. 3, 2015.

[5] Z. Lu, G. Cao, and T. La Porta, "Networking smartphones for disaster recovery," in IEEE PerCom, 2016, pp. 1-9.

[6] A. MartíN-Campillo, J. Crowcroft, E. Yoneki, and R. Martí, "Evaluating opportunistic networks in disaster scenarios," Journal of Network and Computer Applications, vol. 36, no. 2, pp. 870-880, 2013.

[7] V. G. Menon, J. P. Pathrose, and J. Priya, "Ensuring reliable communication in disaster recovery operations with reliable routing technique," Mobile Information Systems, vol. 2016, 2016.

[8] A. Keränen, J. Ott, and T. Kärkkäinen, "The ONE Simulator for DTN Protocol Evaluation," SIMUTools, p. 55, 2009.

[9] N. Aschenbruck, R. Ernst, E. Gerhards-Padilla, and M. Schwamborn, "Bonnmotion: a mobility scenario generation and analysis tool," in SIMUTools'10, 2010, p. 51.

[10] J. T. B. Fajardo, K. Yasumoto, N. Shibata, W. Sun, and M. Ito, "Disaster information collection with opportunistic communication and message aggregation," Journal of information processing, vol. 22, no. 2, pp. 106-117, 2014. 